\title{
Sequential injection titration of chloride in milk with potentiometric detection
}

\author{
M.J. Reis Lima ${ }^{a}$, Sílvia M.V. Fernandes ${ }^{b}$, António O.S.S. Rangel ${ }^{\text {b,* }}$ \\ ${ }^{a}$ Escola Superior Agrária de Viseu, Instituto Superior Politécnico de Viseu, Campus Politécnico, Repeses, 3504-510 Viseu, Portugal \\ ${ }^{\mathrm{b}}$ Escola Superior de Biotecnologia, Universidade Católica Portuguesa, R. Dr. António Bernardino de Almeida, 4200-072 Porto, Portugal
}

Keywords: Sequential injection; Milk; Chloride pseudo-titration

\begin{abstract}
In this work, a sequential injection system for the pseudo-titration of chloride in milk is described. Milk was directly aspirated into the system and sandwiched between two silver nitrate plugs (titrant). The aspirated zones were then propelled to the detector $\left(\mathrm{Ag}_{2} \mathrm{~S} / \mathrm{Ag}\right.$ tubular electrode), where the depletion in the titrant silver concentration (due to the formation of a $\mathrm{AgCl}$ precipitate) was monitored. The results obtained by the developed sequential injection titration method were not statistically different from those provided by the potentiometric reference procedure with relative standard deviations better than $3.4 \%$. The sampling rates achieved were 17 samples per hour.
\end{abstract}

\section{Introduction}

Milk contains many important components such as vitamins, proteins, fatty acids, glucids and salts. The knowledge about the possible physical and chemical combinations of salts in milk is yet incomplete.

Chloride determination is frequently carried out in milk factories. A possible milk adulteration consists of the fraudulent dilution of milk with water, as the payment to the producer is based on milk volume. In order to compensate for milk dilution, fraudulent salt addition is added to milk, to give the same final density. Another possibility causing abnormal values in chloride levels in milk is cow mastitis.

The ubiquitous nature of $\mathrm{Cl}^{-}$and its importance makes its determination one of the most widely studied. The determination of chloride in milk is usually accomplished by titration with silver nitrate. The automation of this determination was previously proposed using the so-called flow injection analysis (FIA) pseudotitrations, with potentiometric detection. In FIA titrations (Ramsing, Ruzicka, \& Hansen, 1981), advantage is

\footnotetext{
${ }^{*}$ Corresponding author. Tel.: +351-225580064; fax: +351225090351

E-mail address: rangel@esb.ucp.pt (A.O.S.S. Rangel).
}

taken from the highly reproducible and controllable dispersion profile of a sample plug (the titrant in the sense of classic volumetry) inserted into a continuous carrier stream (the titrant). The concentration profile (reactant or product), usually created in a well-stirred mixing chamber, is subsequently monitored. An important difference in this technique related to other FIA methods is the use of the measurement of peak width (which corresponds to a time interval) as an alternative to the measurement of the respective peak height. When measurements of the analytical signal are taken at some fixed potential, a linear relationship is obtained between the time interval (peak width) and the logarithm of the analyte concentration (Rhee \& Dasgupta, 1985). Some authors (Ferreira, Lima, \& Rangel, 1994; Lima, Delerue-Matos, \& Vaz, 2000) successfully applied FIA titrations to the determination of chloride in milk.

To overcome some limitations attributed to FIA, sequential injection analysis (SIA) was proposed by Ruzicka and Marshall (1990). Sample and reagent solutions are sequentially aspirated to a holding coil; mixing between sample and reagents occurs during the transport process to the detection system, which involves an inversion of the flow direction. The sequential selection of the various solutions and the subsequent direction to the detector is achieved by the use of a multi-port 
selection valve. Compared to FIA, SIA presents the advantage of lower reagent consumption due to noncontinuous pumping, and has the ability to perform different analysis without system reconfiguration. Although many FIA techniques have been adapted to sequential injection, there are only few works describing titrations in SIA, all dedicated to acid-base titrations (Estela, Mas, Cladera, \& Cerdà, 1999; Holman, Christian, \& Ruzicka, 1997; van Staden \& Plessis, 1997).

Regarding the determination of chloride in milk, a sequential injection system was proposed by Silva, Souza, Ferraz, and Nogueira (1999), using dialysis to separate chloride from the matrix and subsequent conductimetric detection. The aim of our work was to develop a sequential injection system for the determination of chloride in milk based on the same principle of the reference method, a titration with silver cation. Matrix separation becomes unnecessary as a relatively selective reaction is involved, and because of the use of a potentiometric detector, which is not affected by sample colour or turbidity. In the proposed system, instead of using a continuous flow of titrant $\left(\mathrm{AgNO}_{3}\right)$ typical of FIA titrations, the sample was sandwiched between two $\mathrm{AgNO}_{3}$ plugs, being the silver consumption monitored in a laboratory-made $\mathrm{Ag}_{2} \mathrm{~S} / \mathrm{Ag}$ tubular electrode. As in FIA titrations, a linear relationship between the peak width (corresponding to a time interval) and the logarithm of the chloride concentration was achieved.

\section{Materials and methods}

Instrumentation

A Crison 2002 (Barcelona, Spain) digital voltmeter was used for the potentiometric measurements. In the sequential injection determinations, the voltmeter was coupled to a Metrohm E586 labograph (Herisau, Switzerland) chart recorder.

An $\mathrm{Ag}_{2} \mathrm{~S} / \mathrm{Ag}$ tubular electrode (Lima \& Rangel, 1989) was used as indicator electrode; it was prepared from a silver tube supplied by Goodfellow Cambridge Limited (UK), with $1.1 \mathrm{~mm}$ outside diameter and $0.75 \mathrm{~mm}$ inside diameter. The commercial silver cylinder was cut to $1 \mathrm{~cm}$ length and the interior wire of a shielded cable was welded to the fragment obtained, which provided its connection to the voltmeter. Its internal surface was first cleaned by circulating a solution of $0.1 \mathrm{~mol} / 1 \mathrm{HNO}_{3}$ for 5 min. Next, the silver sulphide was deposited in the inner face of the silver tube by circulating a solution of $0.1 \mathrm{~mol} / 1 \mathrm{Na}_{2} \mathrm{~S}$ overnight. Then, the electrode was washed by pumping water and finally conditioned in a $10^{-2}$ $\mathrm{mol} / 1 \mathrm{AgNO}_{3}$ solution.

For the SIA titrations, a double-junction $\mathrm{AgCl} / \mathrm{Ag}$ Orion (Boston, USA) 900200 with a $10 \% \mathrm{KNO}_{3}$ outer filling solution was used as reference electrode.
For the reference procedure, a $\mathrm{Ag}_{2} \mathrm{~S}$ crystalline membrane (Lima \& Rocha, 1990) ion selective electrode was used.

A Gilson Minipuls 3 (Villiers-le-Bel, France) peristaltic pump and PVC Gilson propelling tubes were used for propelling the solutions. The pump was connected to the central channel of an eight port electrically actuated selection valve Valco VICI C15-3118E (Houston, USA). The tubes connecting the different parts of the sequential injection system were made of PTFE Omnifit (Cambridge, UK) with $0.8 \mathrm{~mm}$ i.d.

A 386 personal computer Samsung SD 700 (Korea) equipped with an Advantec PCL 818 L (Taipei, Taiwan) interface card, running a home-made software written in QuickBasic 4.5 (Microsoft) controlled the selection valve positions and the rotation sense and speed of peristaltic pump.

\section{Reagents and solutions}

All solutions were prepared using deionised water with a specific conductivity lower than $0.1 \mu \mathrm{S} / \mathrm{cm}$. All chemicals were of analytical reagent grade.

A chloride stock solution $(1 \mathrm{~mol} / \mathrm{l})$ was prepared by dissolution of $11.69 \mathrm{~g}$ of sodium chloride (previously dried at $110^{\circ} \mathrm{C}$ for $3 \mathrm{~h}$ ) in water, and then quantitatively transferred to a $200 \mathrm{ml}$ volumetric flask. This solution was used to prepare working standards with concentrations between 0.01 and $0.25 \mathrm{~mol} / \mathrm{l}$ of chloride, by rigorous dilution with deionised water.

The $1 \times 10^{-4} \mathrm{~mol} / 1$ silver nitrate solution used as titrant in SIA was prepared by dissolution of $0.0085 \mathrm{~g}$ $\mathrm{AgNO}_{3}$ in a ionic strength adjuster solution composed of $0.5 \mathrm{~mol} / 1 \mathrm{KNO}_{3}$ and $2 \times 10^{-3} \mathrm{~mol} / 1 \mathrm{HNO}_{3}$.

A $1 \times 10^{-7} \mathrm{~mol} / 1 \mathrm{AgNO}_{3}$ solution prepared in the ionic strength adjuster was used as carrier solution.

The silver solution $(0.100 \mathrm{~mol} / \mathrm{l})$ used in the potentiometric titration of the samples by the reference method was standardized against a $\mathrm{NaCl}$ standard solution.

\section{Reference method}

In order to assess the quality of the results obtained applying the developed analytical method, all samples were also analysed by the reference procedure (AOAC, 1997). For the chloride determination in milk, the potentiometric titrimetric method using $0.100 \mathrm{~mol} / \mathrm{l}$ silver (I) solution as titrant is recommended (AOAC, 1997). Before each titration, $30 \mathrm{ml}$ of deionised water and $10 \mathrm{ml}$ of $\mathrm{HNO}_{3} 0.5 \mathrm{~mol} / \mathrm{l}$ were added to the same volume of milk sample.

\section{Sequential injection system}

The developed sequential injection manifold is depicted in Fig. 1. 


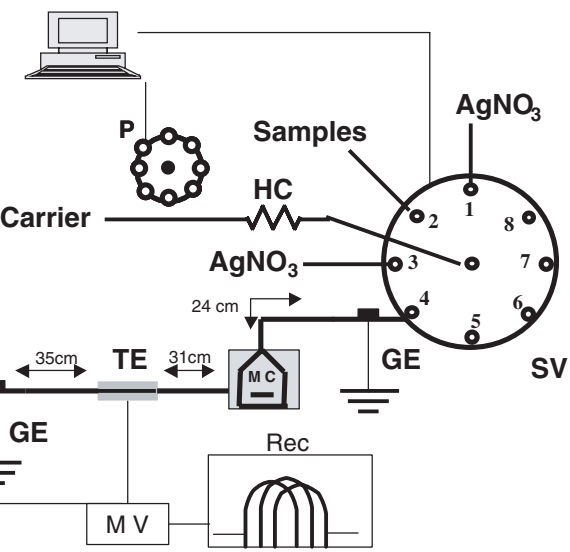

(B) Aspiration sequence for one full cycle

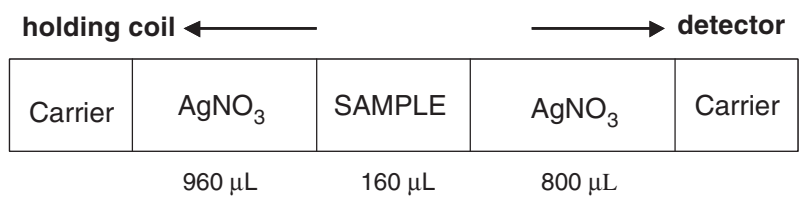

Fig. 1. Sequential injection manifold for the determination of chloride in milk (A) and aspiration sequence for one cycle of the SIA system (B). GE: ground electrode; HC: holding coil; MC: well-stirred mixing chamber; MV: voltmeter; P: peristaltic pump; RE: reference electrode; Rec: chart recorder; SV: selection valve; W: waste; TE: tubular electrode.

Table 1

Protocol sequence and aspiration times for one full cycle of the sequential injection system used for chloride determination in milk

\begin{tabular}{llllll}
\hline Step & Description & Port & Time $(\mathrm{s})$ & Flow rate $(\mathrm{ml} / \mathrm{min})$ & Flow direction \\
\hline $\mathrm{A}$ & Silver nitrate aspiration & 1 & 30 & 1.9 & Reversed \\
B & Aspirate sample or standards & 2 & 5 & 1.9 & Reversed \\
$\mathrm{C}$ & Silver nitrate aspiration & 3 & 25 & 1.9 & Reversed \\
$\mathrm{D}$ & Propelling towards the $\mathrm{Ag}_{2} \mathrm{~S} / \mathrm{Ag}$ electrode & 4 & 150 & 3.8 & Forward \\
\hline
\end{tabular}

The sequence of the steps and the timing sequence is shown in Table 1 . Silver nitrate solution $(960 \mu \mathrm{l})$, standard or sample $(160 \mu \mathrm{l})$ and again silver nitrate solution $(800 \mu \mathrm{l})$, were sequentially aspirated to the holding coil (steps A-C respectively) and then pumped through the well-stirred mixing chamber to the $\mathrm{Ag}_{2} \mathrm{~S} / \mathrm{Ag}$ electrode (step D), where the silver concentration gradient created in the mixing chamber was monitored.

\section{Results and discussion}

\section{Manifold optimisation}

The factors influencing the SIA titrations were studied in a univariant way by performing calibration curves with a serious of chloride standard solutions with concentrations between 0.01 and $0.25 \mathrm{~mol} / \mathrm{l}$. The main variables that affect this type of determination are volumes and reagents concentration, and the type of device used to produce the exponential concentration gradient necessary for peak-width measurements.
Measurements of the peak width (time interval) were taken at three fixed heights (potential values) down from the baseline: 30,38 and $46 \mathrm{mV}$. It was found that the measurements corresponding to $38 \mathrm{mV}$ below the baseline potential provided the best sensitivity.

The use of a tubular shaped electrode rather than a conventional configuration electrode has the advantage of increased mechanical stability, since the incorporation of conventionally shaped units in the system usually leads to noise problems and dead volumes, affecting reproducibility and the return to baseline.

In order to improve the stability of the $\mathrm{Ag}_{2} \mathrm{~S} /$ $\mathrm{Ag}$ tubular electrode baseline, vestigial concentration $\left(1 \times 10^{-7} \mathrm{~mol} / \mathrm{l}\right)$ of silver nitrate was included in the carrier solution.

Silver nitrate concentration of the solutions aspirated in steps $\mathrm{A}$ and $\mathrm{C}$ was studied from $1 \times 10^{-5}$ to $5 \times 10^{-4}$ $\mathrm{mol} / \mathrm{l}$. Sensitivity of the determination strongly increased up to $1 \times 10^{-4} \mathrm{~mol} / \mathrm{l}$ and slightly beyond this concentration. Therefore, the $1 \times 10^{-4} \mathrm{~mol} / 1$ solution was chosen. 
These solutions (carrier and titrant solutions) were both prepared in $0.5 \mathrm{~mol} / 1 \mathrm{KNO}_{3}$ to adjust the ionic strength and to increase the solutions conductivity, and $2 \times 10^{-3} \mathrm{~mol} / 1 \mathrm{HNO}_{3}$ to prevent precipitation of silver oxides and hydroxides.

Concerning the volumes of silver nitrate aspirated in steps A and C, they had to be long enough in order to establish a stable baseline just before and after the peak. This way, one of the factors taken into account was the dispersion of the aspirated zones; the zone that presents less dispersion in the holding coil is the last to be aspirated. This is due to the fact that the residence time of the first aspirated zone in the holding coil, as well as the distance it has to travel to the detector, is much longer than that for the last aspirated zone, resulting in larger dispersion. So, the volumes in steps $\mathrm{A}$ and $\mathrm{C}$ were studied within the range of $640-1280$ and $640-1120 \mu$, respectively.

Regarding the volume used in step $\mathrm{A}$, it was found that it presented little effect on the sensitivity of the determination and a much larger effect in the linear range. It was set to $960 \mu 1$ since this volume was enough to establish a linear relationship within the desired range of concentrations and allowed to obtain a stable baseline after the peak formation. Concerning the volume of silver nitrate used in step C, it was found that it strongly affects sensitivity of the determination. A volume of $800 \mu \mathrm{l}$ was chosen since it provided the best sensitivity and showed to be sufficient for achieving a stable baseline at the beginning of the peak formation.

The sample volume had a marked influence on peak width. It was studied between 64 and $320 \mu$ l. By increasing the sample volume, a larger linear range was attained up to $160 \mu \mathrm{l}$. Beyond this volume, there was no improvement in the linear relationship and sensitivity slightly decreased. Higher sample aspiration volumes would also mean that the silver nitrate aspirated zones had to be increased in order to obtain a stable baseline just before and after the electrode response.

To assess the possibility of carrying out this determination without the use of the mixing chamber, it was replaced by a tube with about the same volume. The calibration curves presented identical slope and intercept values. However, the determination of chloride in milk samples without the mixing chamber led to higher results than the expected according to the batch reference procedure. This can be explained in terms of milk physical properties which caused a delay in the time required to return to baseline when compared to the aqueous standard solutions. This peak broadening leads to an overestimation of the chloride concentration. This way, the use of the mixing chamber showed to be essential because the solutions are homogenised in a perpendicular direction to the flux (Lima \& Rangel, 1989), thus eliminating the influence of physical properties on peak width.
Table 2

Results (mol/l) obtained for the determination of chloride in milk by SIA and reference procedure, and respective relative deviation (RD)

\begin{tabular}{lllc}
\hline Sample & $\begin{array}{l}\text { Reference } \\
\text { procedure }\end{array}$ & SIA & RD (\%) \\
\hline 1 & 0.0220 & 0.0227 & 3.2 \\
2 & 0.0361 & 0.0363 & 0.55 \\
3 & 0.0328 & 0.0329 & 0.31 \\
4 & 0.0258 & 0.0239 & -7.4 \\
5 & 0.0260 & 0.0250 & -3.9 \\
6 & 0.0250 & 0.0249 & -0.40 \\
7 & 0.0268 & 0.0270 & 0.75 \\
8 & 0.0263 & 0.0279 & 6.1 \\
9 & 0.0196 & 0.0187 & -4.6 \\
10 & 0.0265 & 0.0274 & 3.4 \\
11 & 0.0326 & 0.0321 & -1.5 \\
12 & 0.0272 & 0.0293 & 7.7 \\
13 & 0.0271 & 0.0282 & 3.9 \\
14 & 0.0285 & 0.0280 & -1.8 \\
\hline
\end{tabular}

With the optimised system, a typical calibration curve ( $\Delta t$ versus logarithm of concentration) was as follows: $\Delta t=54.6 \log \left[\mathrm{Cl}^{-}\right]+155\left(R^{2}=0.993\right)$.

A complete analytical cycle took about $3.5 \mathrm{~min}$. An analytical cycle is the sum of the time needed for each step plus the time necessary for port selection in the selection valve. Thus, based on the time spent per cycle, the determination frequency was 17 per hour.

\section{Application to milk analysis}

To compare the official and proposed titration method, the chloride content in 14 milk samples was determined by both methods (Table 2).

In every set of SIA determinations, the standards were firstly aspirated to establish the calibration curve and then the samples.

A relation of the type SIA $=C_{0}+S \times($ reference procedure) was established and the results were: $C_{0}=$ $0.0003( \pm 0.0044) \mathrm{mol} / \mathrm{l}$ and $S=1.0161( \pm 0.1595)$, with a correlation coefficient of 0.9414 . The values in parenthesis correspond to the limits of $95 \%$ confidence intervals (Miller \& Miller, 1993). These results show that there is no statistical significant difference between the two sets of results, as slope and correlation coefficient are close to unity and $C_{0}$ value is close to zero.

In order to evaluate the methodology repeatability, the relative standard deviation was calculated from ten consecutive determinations of three different milk samples with concentrations of $0.00923,0.0299$ and 0.0316 $\mathrm{mol} / 1$, being the results $2.8 \%, 2.5 \%$ and $3.4 \%$, respectively.

\section{Conclusions}

The time consuming and labour intensive operations involving the batch titrations consists a real problem to 
an industrial process (van Staden \& Plessis, 1997). The possibility of application of titrations in SIA presents a valorous alternative since this technique is more suitable for process analysis than conventional FIA due to its simplicity and greater degree of automation. In fact, the developed system is fully computerised and can be applied in other kinds of nutrition and animal production studies.

Besides, the reagents consumption is much lower than in FIA titrations. In this respect, the mass of $\mathrm{AgNO}_{3}$ consumed per determination with the developed SIA system is less than a half of the amount consumed with the FIA titration systems. This is particularly important when the reagents involved are expensive and/or pollutants, as in this case.

The use of an $\mathrm{Ag}_{2} \mathrm{~S} / \mathrm{Ag}$ electrode allowed the direct analysis of milk samples without any prior treatment.

The results obtained confirm that the use of a wellstirred mixing chamber is necessary to eliminate the influence of the physical properties of the sample matrix on the transient signal of the tubular electrode.

\section{Acknowledgements}

The authors thank IFADAP for financial support through Project AGRO 273. S.M.V. Fernandes acknowledges financial support from FCT (grant BPD/ 22028/99).

\section{References}

Association of Official Analytical Chemists (AOAC). (1997). Official Methods of Analysis of AOAC International (16th ed.). 3rd revision, Vol. II. Gaithersburg, USA.
Estela, J. M., Mas, F., Cladera, A., \& Cerdà, V. (1999). Study on the implementation of flow titrations in sequential injection analysis. Laboratory Robotics and Automation, 11, 207-216.

Ferreira, I. M. P. L. V., Lima, J. L. F. C., \& Rangel, A. O. S. S. (1994). Flow injection titration of chloride in food products with a silver tubular electrode based on an homogeneous crystalline membrane. Food Chemistry, 50, 423-428.

Holman, D. A., Christian, G. D., \& Ruzicka, J. (1997). Titration without mixing or dilution: Sequential injection of chemical sensing membranes. Analytical Chemistry, 69, 1763-1765.

Lima, J. L. F. C., Delerue-Matos, C., \& Vaz, M. C. V. F. (2000). Potentiometric flow titration with increased sensitive detector for determination of chlorides in milk and dairy products. Ciencia $e$ Tecnologia Alimentar, 2(5), 234-239.

Lima, J. L. F. C., \& Rangel, A. O. S. S. (1989). Chloride pseudotitration in wines by FIA with a $\mathrm{Ag}_{2} / \mathrm{Ag}$ tubular electrode as detector. Journal of Food Composition and Analysis, 2, 356363.

Lima, J. L. F. C., \& Rocha, L. S. M. (1990). FIA tubular potentiometric detectors based on homogeneous crystalline membranes. Their use in the determination of chloride and sulphide ions in water. International Journal of Environmental Analytical Chemistry, 38, 127-133.

Miller, J. C., \& Miller, J. N. (1993). Statistics for analytical chemistry. NewYork: Ellis Horwood.

Ramsing, A. U., Ruzicka, J., \& Hansen, E. H. (1981). The principles and theory of high speed titrations by flow injection analysis. Analytica Chimica Acta, 129, 1-17.

Rhee, J., \& Dasgupta, P. K. (1985). Studies in peak width measurement-based FIA acid-base determinations. Mikrochimica Acta, III, 49-64.

Ruzicka, J., \& Marshall, G. D. (1990). Sequential Injection: A new concept in for chemical sensors process in analysis and laboratory assays. Analytica Chimica Acta, 237, 329-343.

Silva, F. V., Souza, G. B., Ferraz, L. F. M., \& Nogueira, A. R. A. (1999). Determination of chloride in milk using sequential injection automated conductimetry. Food Chemistry, 67, 317322.

van Staden, J. F., \& Hanneli du Plessis (1997). Sequential Injection acid-base titration-a useful application for process analytical science. Analytical Communications, 34, 147-151. 